\title{
Clinical Application of Intracranial Pressure Monitoring Based on Flash Visual Evoked Potential in Treatment of Patients with Hypertensive Intracerebral Hemorrhage
}

\section{Fangping Yu}

Songjiang Hospital Affiliated Shanghai Jiao Tong University https://orcid.org/0000-0002-3678-4981

Yingchun Zhao ( $\sim$ Zhaoyingchun9077@163.com)

Yu Zhang

Songjiang Hospital

Xinghua Luan

Rui Jing Hospital

Ying Wu

Songjiang Hospital

\section{Research article}

Keywords: hypertensive intracerebral hemorrhage, intracranial pressure, flash visual evoked potential, mannitol

Posted Date: September 18th, 2020

DOI: https://doi.org/10.21203/rs.3.rs-29768/v2

License: (1) This work is licensed under a Creative Commons Attribution 4.0 International License. Read Full License 


\section{Abstract}

Objective To investigate the application value of flash visual evoked potential (FVEP) noninvasive intracranial pressure (nICP) monitoring technology in patients with hypertensive intracerebral hemorrhage $(\mathrm{HICH})$.

Methods There were 116 eligible subjects included in the experiment, the final sample size was 102 for this study. They were randomly divided into FVEP nICP monitoring group (experimental group) and the non-monitoring group (control group). The experimental group were examined lumbar puncture immediately after intracranial pressure was monitored by FVEP. Mannitol was used in reducing the elevated intracranial pressure. The serum concentrations of creatinine and urea nitrogen were recorded to assess the renal function. To evaluate the efficacy of FVEP nICP monitoring technique for clinical adjustment of mannitol. The Glasgow prognosis scores (GOS) were evaluated for patients' prognosis between two groups.

Results There was no statistical significance between FVEP nICP measurement and lumbar puncture intracranial pressure measurement ( $195.76 \pm 58.88 \mathrm{mmH} 20$ vs $197.04 \pm 53.72 \mathrm{mmH} 20, \mathrm{P}>0.05)$. Linear correlation analysis indicated that there was a strong positive relationship between the measurements $(r=0.950, P<0.01)$. The duration prescription time and the average usage amount of mannitol in the experimental group was significantly less than that in the control group $(P<0.05)$, and the serum creatinine and urea nitrogen concentrations in the two groups were not statistically significant $(P>0.05)$. The cure rate of the experimental group was higher than that of the control group $\left(\chi^{2}=3.889, P=0.048\right)$.

Conclusion FVEP nICP monitoring technology could replace invasive intracranial pressure monitoring technology in part HICH patients. The application of FVEP nICP technique can reduce the dosage of mannitol and improve the prognosis of patients with $\mathrm{HICH}$.

\section{Background}

Hypertension intracerebral hemorrhage $(\mathrm{HICH})$ with the characteristic of high morbidity, mortality and disability is one of the major diseases that endanger the health of the elderly ${ }^{1}$. High intracranial pressure (ICP) caused by $\mathrm{HICH}$ is a common critical condition in neurology. The elevated ICP leads to the displacement of local brain tissue or the formation of brain herniation, which is the direct cause of the rapid deterioration of patients' condition and even death ${ }^{2}$. Headache, vomiting, and disturbance consciousness are the symptoms of the elevated ICP, but these clinical symptoms are not specific. At present, most of the ICP monitoring methods are invasive. Some limitations of the invasive methods include short-term monitoring, risk of infection and intracranial hemorrhage, restricted mobility of the subject, etc ${ }^{3}$. Therefore, it is necessary to find a noninvasive and reliable monitoring method to replace invasive intracranial pressure (iICP) to help clinical diagnosis and treatment. Flash visual evoked potential (FVEP) has been applied in clinical diagnosis due to its noninvasive and easy to perform ${ }^{4}$. Most clinical studies on FVEP noninvasive intracranial pressure (nICP) monitoring technology focus on 
patients with elevated ICP causd by craniocerebral trauma, subarachnoid hemorrhage and $\mathrm{HICH}^{5,6}$. $\mathrm{HICH}$ is the most common disease of elevated ICP in neurology department. Mannitol is usually used in reducing the elevated ICP, but it can also cause side effects such as renal function damage and electrolyte disorder. In addition, under pathological conditions, mannitol crystals can form a hypertonic state locally through penetrating the damaged blood-brain barrier and aggravate cerebral edema ${ }^{7}$. The absence of published studies showing that management of elevated ICP has an effect on ICH outcome makes the decision whether to monitor and treat elevated ICP unclear in patients with ICH. Here, we monitored the changes of ICP with FVEP nICP monitoring technology in $\mathrm{HICH}$ patients, and evaluate whether the technique is beneficial for clinical practice and reduces complications.

\section{Methods}

\section{Study design and participants}

This study was a prospective registry designed to include consecutive patients with $\mathrm{HICH}$. Only patients with mild to moderate intracerebral hemorrhage were eligible for this study. All subjects were recruited from the emergency department of Neurology, and hospitalized in the department of Neurology and Geriatric department of our hospital from November 2016 to December 2017. All subjects who had a history of hypertension or elevated blood pressure at onset met the diagnostic criteria of the American adult intracerebral hemorrhage treatment guidelines $(2015)^{8}$. They were confirmed by cranial computed tomography (CT) with supratentorial hematoma without or only a small amount of intraventricular hemorrhage, and the amount of hematoma was less than $30 \mathrm{~mL}$ according to the Tada $(A B C / 2)$ formula ${ }^{9}$, with midline shift $<\mathrm{I} \mathrm{cm}$. The vital signs of subjects were relatively stable when they admitted to the hospital. Conservative treatment plan was first adopted after admission with the consent of the family. Exclusion criteria included local infection of the lumber spine, severe liver and kidney dysfunction, pituitary tumor and all diseases associated with damage to visual pathways. Among 116 patients recruited from the emergency department of Neurology, the final sample size was 102 for this study. Because individuals were further excluded for the following reasons: hematoma enlargement requires surgery $(n=9)$, intracranial aneurysm ruptured $(n=2)$, transfer to the superior hospital $(n=2)$, haematuria $(n=1)$. Subjects were randomly divided into FVEP nICP monitoring group (experimental group) and the non-monitoring group (control group). This was in congruence with the local ethics committee requirements. The study was approved by the local ethics committees of our institutions, and subjects informed consent.

\section{Therapeutic methods}

All $\mathrm{HICH}$ patients with elevated ICP were placed in a recumbent position, with elevation of the head of the bed to 30 degrees, kept the airway unobstructed, took sedatives as appropriate to keep the patients calm and kept the surrounding environment quiet, maintained the body temperature below $38.0^{\circ} \mathrm{Cand}$ strictly controlled blood pressure. For HICH patients presenting with SBP between 150 and $220 \mathrm{mmHg}$ and without contraindication to acute BP treatment, acute lowering of the SBP to $140 \mathrm{mmHg}$. For $\mathrm{HICH}$ 
patients presenting with SBP $>220 \mathrm{mmHg}$, antihypertension agents were intermittent or continuous intravenous administration until SBP to $140 \mathrm{mmHg}$. Clinical symptoms and signs were observed every 30 minutes. 20\% mannitol (Sichuan Kelun Pharmaceutical Co.Ltd, Sichun, China, batch no. A17103207-1) was used in reducing the elevated ICP in patients with $\mathrm{HICH}$. Cranial CT was reviewed within $24 \mathrm{~h}$ after admission, and were re-examined at any time according to the changes of the patient's condition. Patients of the experimental group received the first FVEP noninvasive and lumbar puncture iICP measurement within $1 \mathrm{~h}$ after admission. ICP values were monitored by FVEP nICP monitoring device within 24 hours, 3th day, 7th day and 14th day after admission, and mannitol dosage was timely adjusted according to the level of ICP. According to ICP values, the cases were divided into groups as normal (5.0 to $15.0 \mathrm{mmHg}$ ), mildly increased (15.1 to $20.0 \mathrm{mmHg}$ ), moderately increased $(20.1$ to $40.0 \mathrm{mmHg}$ ) and severely increased (more than $40.1 \mathrm{mmHg}$ ). $1 \mathrm{mmHg}$ is converted to $\mathrm{mmH}_{2} \mathrm{O}$ by multiplying with 13.6 . Patients with mildly elevated ICP were observed closely without using mannitol. Patients with moderate elevated ICP lasting more than 10 minutes were offered $125 \mathrm{ml} 20 \%$ mannitol, every 8 hours. The patients with severely elevated ICP were offered $125 \mathrm{ml} 20 \%$ mannitol, every 6 hours. ICP values of patients in the control group were not monitored. The usage amount of mannitol was adjusted according to clinical symptoms, signs, hematoma size shown by CT. Mannitol, $125 \mathrm{ml} 20 \%$ every 6 hours, was used in patients with consciousness disorders and hematoma enlargement by reviewing of cranial CT, and $125 \mathrm{ml} 20 \%$ mannitol, every 8 hours in the other patients. The usage amount of mannitol and renal function were recorded in both groups. All patients were followed up to 3 months after discharge and their GOS scores were recorded.

\section{FVEP non-invasive intracranial pressure monitoring}

FVEP nICP was determined using the MIP-310 nICP monitor (Chongqing Haiweikang Medical Instrument Co. Ltd, Chongqing, China) before treatment within $1 \mathrm{~h}$ after admission. Patients were supine in quiet state, excluding mental factors and environmental interference. The grounding electrode (black line) was placed on the eyebrows, left record electrode (orange line) and the right record electrode (brown line) were respectively placed on the external occipital protuberance $2 \mathrm{~cm}$, and the reference electrode (red line) was placed at the hairline. After wearing the eye mask, the subject was given flash stimulation (the light source was blue neon light with a frequency of $1.0 \mathrm{~Hz}$ and a pulse width of $2 \mathrm{~ms}$ for 50 times). The latency and amplitude of $\mathrm{N} 2$ wave were recorded after the stimulation, then calculated the ICP. With the relation function between $\mathrm{N} 2$ wave and ICP: ICP $=1.0371^{*}$ latency-3.7106 $\left(\mathrm{mmH}_{2} \mathrm{O}\right)$, the ICP values of both left and right channels can be obtained. The testing process should be measured for 3 consecutive times within 15 min for each measurement, and the mean value of 3 times should be taken. The normal ICP range is $80-180 \mathrm{mmH}_{2} \mathrm{O}, \geq 200 \mathrm{mmH}_{2} \mathrm{O}$ means the elevated ICP.

\section{Lumbar puncture}

In our work, cerebrospinal fluid (CSF) lumbar pressure was used in this protocol as a surrogate measurement of ICP. After FVEP nICP monitoring, the lumbar puncture immediately operated in patients of experimental group in order to evaluate the accuracy of the FVEP nICP monitoring values. The patient 
was lying horizontally in the left lateral position. Once the appropriate location was palpated, after local anesthesia a non-traumatic lumbar needle was inserted in the L3-L4 or L4-L5 interspinous space and was left in place during the whole procedure. The CSF pressure was measured with a graduated fluid transducer. The zero reference pressure was the atmospheric pressure at the level of the foramen of Monro. In our study, local infection of the lumber spine and brain herniation were contraindications to lumbar puncture.

\section{Clinical efficacy evaluation}

Prognosis was evaluated using Glasgow Outcome Scale (GOS) standard ${ }^{10}$. level 1: death; Level 2: plant survival state; Level 3: severely disabled, unable to take care of himself; Level 4: mild disability, self-care; Level 5: return to good health and normal life. Level 1 and level 2 were considered invalid, level 3 and level 4 were considered disabled, and level 5 was considered cured. Cure rate $=$ number of patients of level $5 /$ total number of cases $\times 100 \%$.

\section{Statistical analysis}

Statistical analysis was performed using SPSS software (Version 22.0, Chicago, IL, USA). Measurement data was given as mean \pm standard deviation (S.D.) of the mean. Enumeration data was expressed as the count and percentage. Differences between study groups were examined with the $\chi^{2}$-test for categorical variables, and t-test for continuous variables. Pearson correlation analysis was used to analyze the correlation of ICP between the experimental group and the control group. $P<0.05$ was considered statistically significant.

\section{Results}

\section{Study flow chart}

There were 116 eligible subjects included in the experiment, the final sample size was 102 for this study. They were randomly divided into the experimental group $(n=52)$ and the control group $(n=50)$. FVEP nICP monitoring and lumbar puncture test were performed in the experimental group. Cranial CT was examined and renal function was tested in all subjects. In the two groups, different treatment plans were given according to the severity of patients to reduce intracranial pressure. There was no loss of subjects during the experiment (Figure 1).

\section{Status of patients and and outcome of their monitoring}

There were 52 cases in the experimental group including 28 males and 24 females, mean age $61.15 \pm$ 5.84 years. The volume of bleeding was mean $21.16 \pm 4.27 \mathrm{~mL}$ calculated by Multi-field formula. There were 50 cases in the control group including 27 males and 23females, mean age $60.82 \pm 4.18$ years, and the mean bleeding volume was mean $20.73 \pm 5.96 \mathrm{~mL}$. There were no statistically significant differences in the two groups of patients in age, gender and blood loss. The value of FVEP nICP monitoring was 
$195.76 \pm 58.88 \mathrm{mmH} 20$ and the value of lumbar puncture measurement was $197.04 \pm 53.72 \mathrm{mmH} 20$. There was no statistically significant difference $(P>0.05)$.

Then, we used Pearson correlation analysis to analyze the correlation of FVEP nICP monitoring values and lumbar puncture measurement values. The analyses via Pearson's correlation coefficient demonstrated that FVEP nICP monitoring values was positively correlated with lumbar puncture measurement values $(r=0.950)$, the difference was significantly significant $(P<0.01)$ (Figure 2$)$.

\section{Comparison of renal function and mannitol treatment between two groups}

There were two cases who suffered kidney dysfunction in the control group, manifested as elevated creatinine and urea nitrogen values, but no statistical significance was found between the experimental group and the control group $(P>0.05)$. Compared with the control group,

the duration prescription time and the average of mannitol usage in the experimental group was significantly decreased $(P<0.05)$ (Table 1$)$.

Table 1. Comparison of kidney function and mannitol usage between two groups

\begin{tabular}{|l|l|l|}
\hline Variables & experimental group & control group \\
\hline Cr $(\mu \mathrm{mol} / \mathrm{L})$ & $120.38 \pm 5.93$ & $127.83 \pm 6.72$ \\
BUN $(\mathrm{mmol} / \mathrm{L})$ & $7.69 \pm 0.97$ & $8.97 \pm 1.23$ \\
$20 \%$ mannitol usage $(\mathrm{mL})$ & $3509.54 \pm 289.18$ & $4569.25 \pm 308.74 *$ \\
\hline
\end{tabular}

$\mathrm{Cr}$, creatinine; BUN, blood urea nitrogen. Compared to the experimental group, $* P \llbracket 0.05$

\section{Comparison of GOS scores between two groups}

There were 35 patients with GOS grade 5 in the experimental group and 23 patients with GOS grade 5 in the control group. The cure rate in the experimental group was higher than that in the control group $\left(67.3 \%\right.$ vs. $\left.46.0 \%, \chi^{2}=3.889, P=0.048\right)$ (Table 2 ).

Table 2. comparison of GOS scores between the two groups, $\mathrm{n}(\%)$

\begin{tabular}{|c|c|c|c|c|c|}
\hline group & Grade 1 & Grade 2 & Grade 3 & Grade 4 & Grade 5 \\
\hline $\begin{array}{l}\text { experimental group } \\
(\mathrm{n}=52)\end{array}$ & $2(3.8)$ & $3(5.8)$ & $5(9.6)$ & $7(13.5)$ & $35(67.3)$ \\
\hline $\begin{array}{l}\text { control group } \\
(n=50)\end{array}$ & $4(8.0)$ & $6(12.0)$ & $8(16.0)$ & $9(18.0)$ & $23(46.0)$ \\
\hline
\end{tabular}

\section{Discussion}

This study showed that there was no statistically significant difference between the FVEP nICP monitoring values $(195.76 \pm 58.88 \mathrm{mmH} 20)$ and lumbar puncture measurement values $(197.04 \pm 53.72$ 
$\left.\mathrm{mmH}_{2} \mathrm{O}\right)$ in mild to moderate $\mathrm{HICH}$ patients $(P>0.05)$. Pearson correlation analysis demonstrated that FVEP nICP monitoring values was positively correlated with lumbar puncture measurement values, the difference was significant $(r=0.950, P<0.01)$. Through monitoring the change of ICP values by FVEP nICP monitoring techonology, the duration prescription time and the average usage amount of mannitol in the experimental group was significantly less than that in the control group. The complications of kidney function impairment caused by drugs were also rare than those in the control group. Our research also showed that the recovery rate of the experimental group was significantly higher than that of the control group $(67.3 \%$ vs. $46.0 \%, P=0.048)$, and the prognosis of the $\mathrm{HICH}$ patients could be improved by closely monitoring the change of ICP value and timely adopting reasonable treatment plan.

$\mathrm{HICH}$ is an acute and severe disease in department of neurology. Elevated ICP after intracerebral hemorrhage plays an important role in secondary brain injury and is associated with increased mortality 11. Timely detecting of ICP changes is the key to successful rescue of critically ill patients. Nowadays, most methods of ICP monitoring are invasive, but it's more likely to occur intracranial infection, intracranial hemorrhage and other complications ${ }^{12,13,14}$. Mizutani et al. ${ }^{15}$ attempted to evaluate the sizes of intracranial hematoma, subdural hematoma, ventricular, and the degrees of subarachnoid hemorrhage and brain trauma injury through CT imaging, and established the relationship equation between CT imaging and elevated ICP by applying multiple regression analysis. But the results showed that the difference of ICP value was more than $40 \mathrm{mmH}_{2} \mathrm{O}$. Magnetic resonance imaging (MRI) examination is not convenient to be used in critically ill patients, nor can it be used to monitor ICP in a timely and dynamic manner ${ }^{16,17}$. FVEP nICP monitoring technology had been applied in clinical practice since $1986^{18}$. FVEP is the electrical activity generated by the occipital cortex to the visual stimulation induced by the diffuse non-mode light source. The delay time of the second negative wave (N2 wave) of the brain FVEP is directly related to ICP ${ }^{19}$. A microcomputer device can be used to perform visual stimulation and measure the delay time of $\mathrm{N} 2$ wave, then we can obtained the ICP value by comparing the relation table of N2 wave delay time and ICP value ${ }^{20}$. York et al. confirmed in the study of pediatric hydrocephalus and nonopen craniocerebral trauma that there was a strong linear relationship between elevated ICP and prolonged latency of N2 wave of visual evoked potential (the correlation coefficient was 0.8-0.9) ${ }^{19}$. Visual evoked potential is best predicted when cranial hypertension is greater than or equal to $300 \mathrm{mmH}_{2} \mathrm{O}^{21}$.

When a technique is applied to the clinic practice, the first consideration is accuracy. The correlation coefficient between nICP and ICP describes Whether nICP is a good proxy for the ICP. To assess the accuracy of a noninvasive method, the Association of Advancement of Medical Instrumentation stated that when the ICP that ranges between $0 \otimes 20 \mathrm{mmHg}$, a difference of $2 \mathrm{mmHg}$ is acceptable when compared to an invasive method ${ }^{22}$. Our study showed that the difference between the invasive and noninvasive ICP value within $2 \mathrm{mmHg}$. Therefore, we can admit that FVEP nICP monitoring technology is accurate.

Similar to our research, ultrasonographic optic nerve sheath diameter (ONSD) is another noninvasive ICP monitoring technology through detecting optic nerve. Though it is a simple bedside ocular ultrasound 
used in detecting the elevated ICP, the ONSD is moderately correlated with ICP in both right and left eyes respectively and Pearson correlation of ONSD between two eyes (right and left) was 0.749 and $0.726^{23}$. The optimal ONSD cut-off for the identification of ICP has controversial, it fluctuates from $0.48-0.5 \mathrm{~cm}$ 24,25 .

The majority of patients with intracerebral hemorrhage experienced further increase in ICP due to hematoma enlargement within $24 \mathrm{~h}$ after the onset of intracerebral hemorrhage. Some researchers showed that the increase of ICP is significantly earlier than the clinically observed changes in consciousness and vital signs ${ }^{26.27}$. Especially applicable to patients with mild to moderate $\mathrm{HICH}$ without invasive ICP monitoring, FVEP nICP monitoring technology can be used as an effective means of early warning of further increase of ICP and further enlargement of hematoma ${ }^{28}$. Moreover, lumbar puncture manometry has been limited due to contraindications and clinical complications ${ }^{29}$. Therefore, the use of FVEP nICP monitoring is particularly important for $\mathrm{HICH}$ patients, especially for monitoring the change of ICP on the hematoma side and for early evaluating the degree of cerebral hemorrhage and cerebral edema.

In $\mathrm{HICH}$ patients, intracranial hematoma and cerebral edema will lead to intracranial hypertension in $70 \%$ of patients. If not timely intervention will seriously affect the recovery of neurological function and prognosis of patients. Mannitol is the most commonly dehydrating agents which lower ICP by reducing blood viscosity and increasing plasma osmotic pressure ${ }^{30}$. However, clinical usage amount lacks scientific standards and often relies only on clinical experience, as for mannitol to achieve the expected effect is more difficult to determine. In addition, mannitol can cause kidney function damage and electrolyte disturbance. In order to reduce the possible damage caused by using of high doses of mannitol blindly, the American stroke Association recommends that mannitol should not be used prophylactically and should not be used for more than 5 days during first aid ${ }^{31,32}$. Clinical data showed that mannitol could shorten the incubation period of FVEP N2 wave, and FVEP could observe the changes of ICP after mannitol application ${ }^{33}$. In our experiment, the duration prescription time and the average usage amount of mannitol was significantly reduced in the experimental group by applying the FVEP nICP monitoring technology. The ICP changes monitored by FVEP nICP monitoring technology conducive to guide clinical treatment.

\section{Limitations}

Certain limitations of this report need to be acknowledged. Firstly, the study was performed in a small population and in a limited area. There may be a bias in drawing conclusions. Secondly, this study only carried out in mild to moderate $\mathrm{HICH}$ patients, and the application of FVEP nICP monitoring technology in critically ill patients has not been experienced, so further experiments can be attempted.

\section{Conclusions}


Our research showed that the recovery rate of the experimental group was significantly higher than that of the control group, and the prognosis of the $\mathrm{HICH}$ patients could be improved by closely monitoring the change of ICP value and timely adopting reasonable treatment plan. Based on the noninvasive and security of FVEP nICP monitoring technology, it can be considered to be applied to $\mathrm{HICH}$ patients with relatively mild to moderate clinical conditions, good patient compliance, and stability of the disease. The dynamic monitoring of ICP in $\mathrm{HICH}$ patients can guide the clinical timely adjustment of the dosage of mannitol and improve the condition and prognosis of $\mathrm{HICH}$ patients. However, the limitations and influencing factors of FEVP nICP monitoring technology should also be considered during clinical use, so as to better understand its indications and provide more reliable methods and means for clinical treatment of patients with $\mathrm{HICH}$ intracranial hypertension.

\section{Abbreviations}

FVEP: flash visual evoked potential; nICP: noninvasive intracranial pressure; $\mathrm{HICH}$ : hypertensive intracerebral hemorrhage; GOS: glasgow prognosis scores; ICP: intracranial pressure; nICP: noninvasive intracranial pressure; ilCP: invasive intracranial pressure; CT: computed tomography; MRI: magnetic resonance imaging; Cr: creatinine; BUN: blood urea nitrogen.

\section{Declarations}

\section{Ethics approval and consent to participate}

The study was approved by the Ethics Committee of the Songjiang Hospital Affiliated to Shanghai Jiaotong University School of Medicine. Written informed consent was obtained from all participants following a detailed explanation of the study. The study was done in accordance with the principles outlined in the Declaration of Helsinki (1964).

\section{Consent for publication}

Not applicable.

\section{Availability of data and materials}

The data supporting our findings can be found in our article and its additional files.

\section{Competing interests}

The authors declare that they have no competing interests.

\section{Funding}

The OPENS trial is funded by the Shanghai science and technology commission (16411973000). The funder had no involvement in developing the protocol but approved the final submission. 


\section{Authors' contributions}

FP-Y participated in the design of the study, statistical analysis, and drafted the manuscript. YC-Z participated in the design and conduct of the study. Y-Z carried out the operation of the experiment. Y-W provided management and operational assistance to the patients. $\mathrm{XH}-\mathrm{L}$ helped to modify the manuscript. All authors read and approved the final manuscript.

\section{Acknowledgements}

We would like to thank all patients, researchers, and technical assistance by Wenzhong Han to this study. We thank the Shanghai science and technology commission for their invaluable support of this study.

\section{References}

1. Sacco S, Marini C, Toni D, Olivieri L, Carolei A. Incidence and 10 year survival of intracerebral hemorrhage in a population-based registry. Stroke. 2009;40(2):394-399. doi:10.1161/STROKEAHA.108.523209.

2. Davis SM, Broderick J, Hennerici M, Brun NC, Diringer MN, Mayer SA, et al. Hematoma growth is a determinant of mortality and poor outcome after intracerebral hemorrhage. Neurology. 2006;66(8):1175-1181. doi: 10.1212/01.wnl.0000256290.15120.37.

3. Zeng T, Gao L. Management of patients with severe traumatic brain injury guided by intraventricular intracranial pressure monitoring: a report of 136 cases. Chin J Traumatol. 2010;13(3):146-151. doi: 10.3760/cma.j.issn.1008-1275.2010.03.003.

4. Zhao YL, Zhou JY, Zhu GH. Clinical experience with the noninvasive ICP monitoring system.Acta Neurochir Suppl. 2005;95:351-355. doi: 10.1007/3-211-32318-x_72.

5. Qerama E, KorshoejAR, Petersen MV, Brandmeier R, Oettingen GV. Latency-shift of intra-operative visual evoked potential predicts reversible homonymous hemianopia after intra-ventricular meningioma surgery. Clin Neurophysiol Pract. 2019;4:224-229. doi:

10.1016/j.cnp.2019.10.004. eCollection 2019.

6. Wang TZ, Ma S, Guan YC, Du JH, Liu GJ, Zhao XL. Double function of noninvasive intracranial pressure monitoring based on flash visual evoked potentials in unconscious patients with traumatic brain injury. J Clin Neurosci. 2016;27:63-67. doi: 10.1016/j.jocn.2015.08.036.

7. Bereczki D, iu M, Prado GF, Fekete I. Cochrane report\a systematic review of mannitol therapy for acute ischemic stroke and cerebral parenchymal Hemorrhage. Stroke. 2000; 31(11):2719-1722. doi: 10.1161/01.STR.31.11.2719.

8. Hemphill JC 3rd, Greenberg SM, Anderson CS, Becker K, Bendok BR, Cushman M, et a1 $₫$ Guidelines for the management of spontaneous intracerebral hemorrhage $\ a$ guideline for healtheare professionals from the American Heart Association/American Stroke Association. Stroke. 2015;46(7):2032-2060. doi: 10.1161/STR.0000000000000069. 
9. Kothari RU, Brott T, Broderick JP, Barsan WG, Sauerbeck LR, Zuccarello M, et al. The ABCs of measuring intracerebral hemorrhage volumes. Stroke. 1996;27:1304-1305. doi囚 10.1161/01.STR.27.8.1304

10. Gross T, Morell S, Amsler F. Gender-Specific Improvements in Outcome 1 and 2 Years After Major Trauma. J Surg Res. 2019; 235:459-469. doi: 10.1016/j.jss.2018.10.024.

11. Badri S, Chen J, Barber J, Temkin NR, Dikmen SS, Chesnut RM, et al. Mortality and long-term functional outcome associated with intracranial pressure after traumatic brain injury. Intensive Care Med. 2012;38(11): 1800-1809. doi: 10.1007/s00134-012-2655-4.

12. Collins CD, Hartley JC, Chakraborty A, Thompson DN. Long subcutaneous tunneling reduces infection rates in paediatric external ventricular drains. Childs Nerv Syst. 2014; 30(10):1671-1678. doi: 10.1007/s00381-014-2523-3.

13. Lescot T, Reina V, Le Manach Y, Boroli F, Chauvet D, Boch, AL, at al. In vivo accuracy of two intraparenchymal intracranial pressure monitors. Intensive Care Med. 2011;37(5):875-879. doi: 10.1007/s00134-011-2182-8.

14. Raboel PH, Bartek J, Jr, Andresen M, Bellander BM, Romner B. Intracranial pressure monitoring: invasive versus non-invasive methods-a review. Crit Care Res Pract. 2012;2012:950393. doi: 10.1155/2012/950393.

15. Mizutani T, Manaka S, Tsutsumi H. Estimation of intracranial pressure using computed tomography scan findings in patients with severe head injury. Surg Neurol. 1990;33(3):178-84. doi: 10.1016/00903019(90)90181-N.

16. Kristiansson H, Nissborg E, Bartek J Jr, Andresen M, Reinstrup P, Romner B. Measuring elevated intracranial pressure through noninvasive methods: a review of the literature. $J$ Neurosurg Anesthesiol. 2013;25(4):372-385. doi: 10.1097/ANA.0b013e31829795ce.

17. Alperin NJ, Lee SH, Loth F, Raksin PB, Lichtor T. MR-Intracranial pressure (ICP): a method to measure intracranial elastance and pressure noninvasively by means of MR imaging: baboon and human study. Radiology. 2000;217:877-85. doi: 10.1148/radiology.217.3.r00dc42877.

18. Rosenield JG『Method and appratus for intracranial pressure estiationखU.S. Patent 4564022[P], 1986囚 $1 \otimes 14$.

19. York DH, Pulliam MW, Rosenfeld JG, Watts C. Relationship between visual evoked potentials and intracranial pressure. J Neurosurg. 1981;55(6):909-916. doi: 10.3171/jns.1981.55.6.0909.

20. LENHARDT MARTIN, L. Non-invasive cerebral spinal fluid pressure monitor apparatus and method. United States patent US 973992. 2003 Oct 23.

21. York $D$, Legan $M$, Benner $S$, Watts $C$. Further studies with a noninvasive method of intracranial pressure estimation. Neurosurgery. 1984;14(4):456-461. doi:10.1097/00006123-198404000-00011.

22. The Brain Trauma Foundation. The American Association of Neurological Surgeons. The Joint Section on Neurotrauma and Critical Care. Recommendations for intracranial pressure monitoring technology. J Neurotrama. 2000;17(6_7):497-506. Doi:10.1089/neu.2000.17.497. 
23. Ali MA, Hashmi M, Shamim S, Salam B, Siraj S, Salim B. Correlation of optic nerve sheath diameter with direct measurement of intracranial pressure through an external ventricular drain. Cureus. 2019 Sep 26;11(9):e5777. doi: 10.7759/cureus.5777.

24. RajajeeV, Vanaman M, Fletcher JJ, Jacobs TE. Optic nerve ultrasound for the detection of raised intracranial pressure. Neurocrit Care. 2011 Dec;15(3):506-15. doi: 10.1007/s12028-011-9606-8.

25. Kim SE, Hong EP, Kim HC, Lee SU, Jeon JP. Ultrasonographic optic nerve sheath diameter to detect increased intracranial pressure in adults: a meta-analysis. Acta Radiol. 2019 Feb;60(2):221-229. doi: $10.1177 / 0284185118776501$.

26. Yu SX, Zhang QS, Yin Y, Liu Z, Wu JM, Yang MX. Continuous monitoring of intracranial pressure for prediction of postoperative complications of hypertensive intracerebral hemorrhage. Eur Rev Med Pharmacol Sci. 2016;20(22):4750-4755.PubMed PMID: 27906426. PubMed Central PMCID: PMC2653214.

27. Marcolini E, Stretz C, DeWitt KM. Intracranial Hemorrhage and Intracranial Hypertension. Emergency Medicine Clinics of North America. 2019;37(3), 529-544. doi:10.1016/j.emc.2019.04.001.

28. Wang TZ, Ma S, Guan YC, Du JH, Liu GJ, Zhao XL. Double function of noninvasive intracranial pressure monitoring based on flash visual evoked potentials in unconscious patients with traumatic brain injury. J Clin Neurosci. 2016 May;27:63-67. doi: 10.1016/j.jocn.2015.08.036.

29. Zhang X, Medow JE, Iskandar BJ, Wang F, Shokoueinejad M, Koueik J, et al. Invasive and noninvasive means of measuring intracranial pressure: a review. Physiol Meas. $2017 \mathrm{Jul}$ 24;38(8):R143-R182. doi: 10.1088/1361-6579/aa7256.

30. James HE. The effect of intravenous fluid replacement on the response to mannitol in experimental cerebral edema: an analysis of intracranial pressure, serum osmolality, serum electrolytes, and brain water content. Acta Neurochir Suppl. 2006;96:125-129. doi: 10.1007/3-211-30714-1_28.

31. Fink ME. Osmotherapy for intracranial hypertension: mannitol versus hypertonic saline. Continuum( Minneap Minn). 2012;18(3):640-654. doi: 10.1212/01.CON.0000415432.84147.

32. Thomassen $L \otimes B$ Bainin $M \otimes D$ Demarin $V$, Grond M, Toni D, Venables GS. Acute stroke treatment in Europe: a questionnaire-based survey on behalf of the EFNS Task Force on acute neurological stroke care. Eur J Neurol. 2003;10(3):199-204. doi: 10.3760/cma.j.issn.1673-4165.2004.02.001.

33. Zhou ZJ, Zheng J, Zhao SF, Li QN. The study of the effect of the administration of mannitol on flash visual evoked potentials. Zhonghua Yi Xue Za Zhi. 2005 Oct 12;85(38):2696-9. doi: 10.3760/j:issn:0376-2491.2005.38.008 